\title{
Measuring ambiguity attitude: (Extended) multiplier preferences for the American and the Dutch population
}

\author{
Aurélien Baillon $^{1}$ • Han Bleichrodt ${ }^{1}$. \\ Zhenxing Huang ${ }^{2,3} \cdot$ Rogier Potter van Loon ${ }^{1}$
}

\begin{abstract}
Empirical studies of ambiguity aversion often use measures that are not grounded in theory. This paper shows how a theoretically-founded measure of ambiguity aversion can be derived from Hansen and Sargent's theory of multiplier preferences. Multiplier preferences are used in macroeconomics to capture model uncertainty. At the micro level, they have not been applied yet, because they do not permit ambiguity seeking, which is usually observed for a substantial proportion of subjects. We give a preference foundation for (extended) multiplier preferences accommodating both ambiguity aversion and ambiguity seeking and we propose a simple method to measure them using matching probabilities. We illustrate our method in two large representative samples (Dutch and American) and obtain the first micro estimates of multiplier preferences.
\end{abstract}

Keywords Ambiguity $\cdot$ Multiplier preferences $\cdot$ Robustness $\cdot$ Measurement

Han Bleichrodt

bleichrodt@ese.eur.nl

Aurélien Baillon

baillon@ese.eur.nl

Zhenxing Huang

ybhzxadam@gmail.com

Rogier Potter van Loon

pottervanloon@ese.eur.nl

1 Erasmus School of Economics, Erasmus University Rotterdam, P.O. Box 1738, 3000DR Rotterdam, the Netherlands

2 School of Economics, Shanghai University of Finance and Economics, 111 Wuchuan Rd, Shanghai, China

3 The Key Laboratory of Mathematical Economics (SUFE), Ministry of Education, 111 Wuchuan $\mathrm{Rd}$, Shanghai, China 


\section{JEL Classification D81}

While both the theoretical and the empirical literature on ambiguity are rich, ${ }^{1}$ there is only limited interaction between the two. An important reason is that most ambiguity models use concepts that are hard if not impossible to observe empirically. Most empirical measurements of ambiguity have therefore resorted to pragmatic measures that lack a foundation in theory. ${ }^{2}$ The purpose of this paper is to provide a bridge between theory and empirics. We use Hansen and Sargent's (2001) multiplier preferences model, which captures ambiguity aversion by a single parameter, to derive a theoretically-founded measure of ambiguity aversion. We extend the multiplier preference model to capture all kinds of ambiguity attitudes, we present a method to measure the ambiguity parameter, and we apply our method in two large representative surveys.

Multiplier preferences are widely used in macroeconomics and finance to permit that decision makers' beliefs about economic phenomena are non-unique. In the multiplier preferences model, decision makers rank payoff profiles $f$ according to the criterion:

$$
V(f)=\min _{p} \int u(f) d p+\frac{1}{\sigma} R(p \| q)
$$

where $u$ is a utility function, $q$ is a subjective probability distribution on the states of nature, $\sigma$ is a behavioral parameter, and $R(p|| q)$ is the relative entropy of any probability distribution $p$ with respect to $q$. The intuition underlying Eq. (1) is that the decision maker has some best guess $q$ of the probability distribution on the states of nature, but he does not have full confidence in his guess and also considers other probability distributions $p$. The plausibility of these other distributions decreases with their divergence from $q$, as measured by the relative entropy $R$. The parameter $\frac{1}{\sigma}$ captures the degree to which the decision maker takes alternative probability distributions into account. The lower is $\sigma$, the more the decision maker trusts that $q$ is the correct distribution. In the limit, if $\sigma$ goes to zero, Eq. (1) becomes subjective expected utility.

The lack of trust decision makers have in their beliefs may result from ambiguity (Hansen and Sargent 2001). In empirical studies, most subjects are not neutral towards ambiguity, as assumed by expected utility, but are ambiguity averse. Multiplier preferences capture ambiguity aversion (by the parameter $\sigma$ ) while remaining analytically convenient and easy to incorporate in economic models of aggregate behavior. However, they do not accommodate ambiguity seeking, which limits their applicability at the micro level where a wide range of ambiguity attitudes is typically observed and a substantial proportion of respondents is ambiguity seeking (Trautmann and van de Kuilen 2015).

This paper extends multiplier preferences to accommodate both ambiguity aversion and ambiguity seeking. We give a preference foundation of this extended model that complements Strzalecki (2011) and that makes multiplier preferences suitable for microeconomic applications.

We then present a simple method to measure extended multiplier preferences. Our method is easy to apply and measures multiplier preferences at the individual subject level. Hence, we obtain an axiomatically founded measure of ambiguity aversion that

\footnotetext{
${ }^{1}$ See Trautmann and van de Kuilen (2015) for a recent survey of the empirical literature and Gilboa and Marinacci (2013) or Machina and Siniscalchi (2014) for surveys of the theoretical literature.

${ }^{2}$ Exceptions are Dimmock et al. $(2015,2016 b)$.
} 
can easily be used in empirical research and that captures the heterogeneity in individual ambiguity attitudes.

We illustrate our method using two large representative samples, collected by Dimmock et al. $(2015,2016 \mathrm{a}, \mathrm{b})$ of the Dutch and the US population involving over 5000 subjects in total and we provide the first micro estimates of (extended) multiplier preferences. Most subjects were moderately ambiguity averse, but between 23\% (Dutch sample) and 36\% (US sample) were ambiguity seeking. In both samples, we observed that education and income were negatively correlated with the deviation from ambiguity neutrality. In other words, respondents with more education or a higher income were less likely to deviate from subjective expected utility.

The next section introduces the extended multiplier preferences model and Section 2 its axiomatization. We then show in Section 3 how extended multiplier preferences can be measured and present the empirical results. Section 4 concludes. All proofs are in the Appendix.

\section{Extended multiplier preferences}

We use the Anscombe-Aumann setting. Let $S$ be the state space, i.e. the set of all possible states of nature $s . S$ can be finite or infinite. One state $s$ will occur but the decision maker does not know which one. $\Sigma$ denotes a sigma-algebra on $S$. Its elements are called events and are typically denoted $E$. The set of all countably additive probability measures on $(S, \Sigma)$ is denoted by $\Delta(S)$ and is endowed with the weak* topology. A probability measure $p \in \Delta(S)$ is absolutely continuous with respect to $q \in \Delta(S)$ if for all $E \in \Sigma, q(E)=0$ implies $p(E)=0$. Let $\Delta(q)$ denote the set of all countably additive probability measures that are absolutely continuous with respect to $q$. For any $p, q \in \Delta(S)$, the relative entropy of $p$ with respect to $q$ is given by $R(p \| q)=\int_{S} \log \left(\frac{d p}{d q}\right) d p$ if $p \in \Delta(q)$ and $R(p \| q)=\infty$ otherwise.

We denote the outcome set by $Z . \Delta(Z)$ is the set of all simple lotteries on $Z$. Elements of $\Delta(Z)$ are denoted as $\mathrm{x}, y$. The decision maker chooses between acts, finite-valued mappings from $S$ to $\Delta(Z)$, which are $\Sigma$-measurable. Acts are denoted $f, g$. For event $E, f_{E} g$ denotes the act that gives $f(s)$ if $s \in E$ and $g(s)$ if $s \in E^{c}$ with $E^{c}$ the complement of $E$. The set of all acts is $\mathcal{F}$. Acts have two stages: the first stage corresponds to the uncertainty modeled by $S$ and the second stage to the risks modeled by $\Delta(Z)$. The mixture act $\alpha f+(1-\alpha) g$ for $\alpha \in[0,1]$ is the act that assigns the lottery $\alpha f(s)+(1-\alpha) g(s)$ to state $s$ for all $s \in S$. The decision maker's preferences over acts in $\mathcal{F}$ are denoted by $\succcurlyeq$ (with $\sim, \succ$, $\preccurlyeq$, and $\prec$ defined as usual). A functional V represents $\succcurlyeq$ if $V: \mathcal{F} \rightarrow \mathbb{R}$ is such that $f \succcurlyeq g \Leftrightarrow V(f) \geq V(g)$.

Definition 1: We call $\succcurlyeq$ extended multiplier preferences if $\succcurlyeq$ can be represented by

$$
V(f)=\left\{\begin{array}{cc}
\min _{p \in \Delta(S)} \int_{S} u(f(s)) d p(s)+\frac{1}{\sigma} R(p \| q) & \text { if } \sigma>0 \\
\int_{S} u(f(s)) d q(s) & \text { if } \sigma=0 \\
\max _{p \in \Delta(S)} \int_{S} u(f(s)) d p(s)+\frac{1}{\sigma} R(p \| q) & \text { if } \sigma<0
\end{array}\right.
$$

where $u$ is a nonconstant expected utility functional, $q \in \Delta(S)$, and $\sigma \in \mathbb{R}$. We call these preferences robust if $\sigma \geq 0$ and opportunity seeking if $\sigma \leq 0$. 
The novelty of Definition 1 is that we also consider decision makers who are opportunity seeking $(\sigma \leq 0)$. Previous representations only considered decision makers with robust preferences $(\sigma \geq 0)$.

In contrast with a decision maker with robust preferences, who tries to find options that are maximally insensitive to remaining uncertainties, an opportunity-seeking decision maker values options for which the remaining uncertainties can lead to high expected utilities. He chooses the act that maximizes the highest expected utility he may reach minus a cost, which depends on the divergence between the probability measures $p$ used to compute his expected utility and his best guess $q$. The parameter $\sigma$ indicates to what extent the opportunity-seeking decision maker is constrained in his search to maximize expected utility. The more negative is $\sigma$, the lower the cost of taking account of probability distributions which deviate from his best guess. In the limit, if $\sigma$ goes to minus infinity the decision maker ignores this cost and chooses the probability distribution that maximizes his expected utility, regardless how far off it is from his best guess.

An alternative interpretation of the extended multiplier preferences approach comes from a comparison with $\int u(f) d p+\theta[R(p|| q)-\eta]$, the Lagrange function deduced from minimizing (in the robust approach) or maximizing (in the opportunity seeking approach) $\int u(f) d p$ such that the relative entropy does not exceed a threshold $(R(p|| q)<\eta)$. This comparison shows that the multiplier parameter $\theta=\frac{1}{\sigma}$ is the Lagrange multiplier of the optimization problem and can be interpreted as the shadow price of relaxing the constraint imposed on the relative entropy (Hansen and Sargent 2001).

The parameter $\sigma$ can be interpreted as an index of ambiguity aversion. Lemma A1 in the Appendix shows that extended multiplier preferences are ordinally equivalent to second-order expected utility (SOEU). ${ }^{3}$

$$
V(f)=\int_{S} \varphi_{\sigma}(u(f(s))) d q(s)
$$

when $\varphi_{\sigma}$ is exponential:

$$
\varphi_{\sigma}(t)=\left\{\begin{array}{cc}
-e^{-\sigma t} & \text { if } \sigma>0 \\
t & \text { if } \sigma=0 \\
e^{-\sigma t} & \text { if } \sigma<0
\end{array}\right.
$$

and $u, q$, and $\sigma$ are as in Definition 1. Axiomatizations of SOEU were given by Grant et al. (2009), Nau (2006), Neilson (2010), and Cerreia-Vioglio et al. (2012). Notice that in SOEU as we define it here and as it is also defined by Strzalecki (2011), $q$ is defined over events and not over probability distributions as for example in the smooth model of Klibanoff et al. (2005).

We know from Pratt (1964) that under expected utility the exponential utility function is equivalent to constant absolute risk aversion. This implies that adding an amount $c$ to all outcomes of the lotteries under comparison does not change the preferences between these lotteries. For the exponential function, the Arrow-Pratt index of risk attitude $-\frac{u^{\prime \prime}}{u^{\prime}}$ is constant and equal to the exponential parameter. Under SOEU, we can give a similar interpretation

\footnotetext{
${ }^{3}$ SOEU shows that ambiguity attitudes can be modeled by relaxing the assumption of reduction of compound lotteries between the objective stage (the lottery $f(s)$ ) and the subjective stage (the subjective probability $q(s)$ ). Segal (1987) first made this point using rank-dependent utility in both stages. Dillenberger and Segal (2015) showed that Segal's model also accommodates examples of ambiguity behavior proposed by Machina (2009, 2014) that most other ambiguity models cannot accommodate.
} 
to the exponential $\varphi_{\sigma}$ function in terms of utility: adding the same (expected) utility to each state of the acts under comparison does not change the preferences between these acts. Grant and Polak (2013) describe this property as constant absolute uncertainty aversion.

The index $-\frac{\varphi^{\prime \prime}}{\varphi^{\prime}}=\sigma$ is then an Arrow-Pratt index of ambiguity attitude. ${ }^{4}$

Because extended multiplier preferences have only one parameter more than expected utility, it cannot disentangle ambiguity aversion and ambiguity perception (unlike, for instance, the model used by Dimmock et al. 2015). As we explained above, the parameter $\sigma$ can be seen as an index of ambiguity aversion, but larger absolute values of $\sigma$ also imply that the decision maker considers a larger set of probability distributions and, in that sense, $\sigma$ can also be taken as an index of ambiguity perception.

\section{Axiomatization}

Strzalecki (2011) axiomatized extended multiplier preferences for $\sigma \geq 0$, i.e. for decision makers with robust preferences. We will characterize extended multiplier preferences in full, i.e. including the case of opportunity seeking $(\sigma \leq 0)$. We do so by dropping uncertainty aversion (his A.5) from Strzalecki's set of axioms and by replacing the results in his proof that depend on this axiom by others that do not depend on it.

We impose the following conditions on $\succcurlyeq$ :

1. Weak order: $\geqslant$ is complete and transitive.

2. Weak certainty independence: for all $f, g \in \mathcal{F}$, for all $x, y \in \Delta(Z)$, and for all $\alpha \in$ $(0,1), \alpha f+(1-\alpha) x \geqslant \alpha g+(1-\alpha) x \Rightarrow \alpha f+(1-\alpha) y \geqslant \alpha g+(1-\alpha) y$.

3. Continuity: for all $f, g, h \in \mathcal{F}$, the sets $\{\alpha \in[0,1]: \alpha f+(1-\alpha) g \succcurlyeq h\}$ and $\{\alpha \in[0$, 1] : $\alpha f+(1-\alpha) \mathrm{g} \preccurlyeq h\}$ are closed.

4. Monotonicity: for all $f, g \in \mathcal{F}$ if $f(s) \geqslant g(s)$ for all $s \in S$ then $f \geqslant g$.

5. Nondegeneracy: there exist acts $f, g \in \mathcal{F}$ such that $f \succ g$.

6. Weak monotone continuity: for all $f, g \in \mathcal{F}$, for all $x \in \Delta(Z)$, and for all $\left\{E_{n}\right\}_{n \geq 1} \in \Sigma$ with $E_{1} \supseteq E_{2} \ldots$ and $\cap_{n \geq 1} E_{n}=\varnothing, f \succ g$ implies that there exists an $n_{0}$ such that $x_{E_{n_{0}}} f \succ g$.

7. Sure thing principle: for all $E \in \Sigma$ and for all $f, g, h, h^{\prime} \in \mathcal{F}, f_{E} h \geqslant g_{E} h \Rightarrow f_{E} h^{\prime} \geqslant g_{E} h^{\prime}$.

An event is essential if there exist $f, g, h \in \mathcal{F}$ such that $f_{E} h \succ g_{E} h$.

Theorem 1: If $S$ has at least three disjoint essential events ${ }^{5}$ then the following two statements are equivalent:

1. $\succcurlyeq$ is a continuous, nondegenerate weak order that satisfies weak certainty independence, monotonicity, weak monotone continuity and the sure thing principle.

2. $\succcurlyeq$ has an extended multiplier representation.

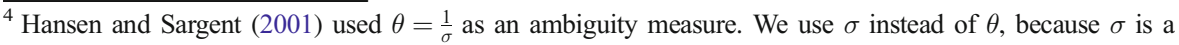
monotonic and continuous measure and, therefore, more convenient for statistical analysis.

${ }^{5}$ If only one event is essential then the Theorem also holds but the uniqueness properties are different. If exactly two disjoint events are essential then the sure thing principle should be strengthened to the hexagon condition (Wakker 1989).
} 
Observation 1: Two triples $(\sigma, u, q)$ and $\left(\sigma^{\prime}, u^{\prime}, q^{\prime}\right)$ represent the same extended multiplier preference if and only if $q$ and $q^{\prime}$ are identical and there exist $\alpha>0$ and $\beta \in \mathbb{R}$ such that $u^{\prime}=\alpha u+\beta$ and $\sigma^{\prime}=\sigma / \alpha$.

We can distinguish the robust and the opportunity seeking approaches using Schmeidler's (1989) condition of ambiguity aversion and its counterpart of ambiguity seeking.

Definition 2: Ambiguity aversion (seeking) holds if for all acts $f, g$ in $\mathcal{F}$ and for all $\alpha$ in $(0,1), f \sim g \Rightarrow \alpha f+(1-\alpha) g \succcurlyeq(\preccurlyeq) f$.

Theorem 2: Under extended multiplier preferences, ambiguity aversion is equivalent to robust preferences and ambiguity seeking is equivalent to opportunity seeking preferences.

According to Theorem 2, the sign of $\sigma$ determines whether an agent is ambiguity averse or ambiguity seeking. But for $\sigma$ to be a proper index of ambiguity aversion, it should also satisfy the property that a higher value represents more ambiguity aversion. We will show that this is indeed the case. Consider two decision makers $i \in\{1,2\}$ represented by preferences $\succcurlyeq_{i}$. We use the definition of "more ambiguity averse" proposed by Ghirardato and Marinacci (2002).

Definition 3: $\succcurlyeq_{2}$ is more ambiguity averse than $\succcurlyeq_{1}$ if for all acts $f$ in $\mathcal{F}$ and lotteries $x$ in $\Delta(Z), x \succcurlyeq_{1} f \Rightarrow x \succcurlyeq_{2} f$.

This definition adapts the definition of "more risk averse" introduced by Yaari (1969) to ambiguity. It implies that the ambiguity attitudes of two decision makers can only be compared if they share the same beliefs (here, the same $q$ ). Moreover, as shown by Ghirardato and Marinacci (2002, Proposition 11), the decision makers need to have the same risk attitudes, which implies that their utility functions must be cardinally equivalent: $u_{1} \approx u_{2}$ if there exist $\alpha>0$ and $\beta \in \mathbb{R}$ such that $u_{1}=\alpha u_{2}+\beta$.

Theorem 3: Given two extended multiplier preferences $\succcurlyeq_{1}$ and $\succcurlyeq_{2}$ represented by $\left(\sigma_{1}, u_{1}, q_{1}\right)$ and $\left(\sigma_{2}, u_{2}, q_{2}\right)$, the following two statements are equivalent:

1. $\succcurlyeq_{2}$ is more ambiguity averse than $\succcurlyeq_{1}$.

2. $u_{1} \approx u_{2}, q_{1}=q_{2}$, and $\sigma_{1} \leq \sigma_{2}$ (if we scale utility such that $u_{1}=u_{2}$ ).

Theorem 3 shows that $\sigma$ is a proper measure of ambiguity aversion.

\section{Measuring extended multiplier preferences}

\subsection{Method}

Strzalecki (2011, Example 3) explained how the multiplier parameter $\sigma$ could be measured when utility $u$ is a power function. We describe an alternative method that makes no assumptions about utility and requires fewer questions. Our method is easier to explain using SOEU with $\varphi_{\sigma}$ exponential and we will, therefore, use this model in what follows. Because extended multiplier preferences are ordinally equivalent to $\operatorname{SOEU}$ with $\varphi_{\sigma}$ exponential, all results remain valid under extended multiplier preferences. 
Suppose that a ball is drawn from an Ellsberg urn with an unknown number of yellow and purple balls (Ellsberg 1961). Let $S=\{Y, P\}$ where $Y$ stands for "the ball is yellow" and $P$ for "the ball is purple". The decision maker can win either $\$ 15$ or nothing, depending on the color of the ball. Hence, $Z=\{0,15\}$. The act $f_{Y}$ pays $\$ 15$ if the ball is yellow and nothing otherwise and the act $f_{P}$ pays $\$ 15$ if the ball is purple and nothing otherwise. Each lottery from $\Delta(Z)$ can be written as $15_{r} 0$, where $r$ is the probability to get 15. We scale utility so that $u(0)=0$ and $u(15)=15$. Then $u\left(15_{r} 0\right)=r^{*} 15+(1-r)^{*} 0=15 r$.

Assume $f_{Y} \sim f_{P} \sim 15_{r} 0$ for some probability $r$. We call this probability $r$ a matching probability of the acts $f_{Y}$ and $f_{P}$. Under SOEU, $f_{Y} \sim f_{P}$ implies $q(Y)=q(P)=1 / 2$. In the multiplier preferences model, this means that the decision maker's best guess is that $Y$ and $P$ are equally likely. The second indifference, $f_{P} \sim 15_{r} 0$, then implies $\varphi_{\sigma}(15 r)=1 / 2 \varphi_{\sigma}(15)+$ $1 / 2 \varphi \sigma(0)$. This equation has a unique solution $\sigma$ for each value of $r \in(0,1)$ (see proof in the Appendix). If $r=1 / 2$, then $\sigma=0$ and the decision maker is indifferent between an objective and a subjective probability of $1 / 2$. This corresponds to ambiguity neutrality. If $r$ $<1 / 2$ then $\sigma>0$ and the decision maker prefers an objective probability of $1 / 2$ to a subjective probability of $1 / 2$. This corresponds to ambiguity aversion. Finally, $r>1 / 2$ implies ambiguity seeking $(\sigma<0){ }^{6}$ If $r \rightarrow 0$, preferences are extremely robust (ambiguity averse) and $\sigma \rightarrow+\infty$. If $r \rightarrow 1$, preferences are extremely opportunity seeking and $\sigma \rightarrow-\infty$.

In the example above and in what follows, we use Ellsberg urns. The elicitation method can be adapted to any naturally occurring complementary events $Y$ and $P$ for which $f_{Y} \sim f_{P}$ The Ellsberg urn offers readily available events that satisfy these conditions.

\subsection{Calibration}

Observation 1 shows that the sign of the multiplier parameter does not depend on the scaling of the utility function, but its magnitude does. Hence, the scaling should be the same for all subjects to perform correlation analysis. Fortunately, the scaling of the utility function can be arbitrary under expected utility, because it does not affect the curvature of utility and thus, risk aversion. In the empirical study reported in Section 3.3, we scale utility of all subjects such that the utility of initial wealth $W$ is 0 and that of $W+15$ is 15 .

Beyond the issue of scaling, our method requires no knowledge of utility. We can immediately measure extended multiplier preferences for ambiguity without having to measure utility.

\subsection{Empirical illustration}

Two surveys have been held in which subjects answered questions of the form described in Section 3.1. Dimmock et al. (2016b) ran a survey among 1900 participants of the Dutch Longitudinal Internet Study for the Social Sciences (LISS). Dimmock et al. (2015, 2016a) ran a similar survey among 3300 participants of the American Life Panel (ALP). ${ }^{7}$ We illustrate our method by showing the $\sigma$ values obtained from these two datasets.

\footnotetext{
${ }^{6}$ For an early application of using matching probabilities to measure ambiguity aversion see Viscusi and Magat (1992).

7 These papers analyzed a subset of their respondents, excluding subjects who took too much or too little time in answering. For example, Dimmock et al. (2016b) excluded more than half of their subjects as these were not incentivized. In our analyses, we chose to include all subjects as any exclusion criterion is to some extent arbitrary. The numbers of observations we present are thus not identical to those mentioned in Dimmock et al. $(2015,2016 a, b)$, but our results are practically unaffected by the inclusion criteria.
} 
In both surveys, subjects had to choose between two urns: a known urn $\mathrm{K}$ and an ambiguous urn A. Urn K contained 100 yellow and purple balls in known proportions. Urn A contained 100 yellow and purple balls in unknown proportions. By default, purple was the winning color, but subjects could change the winning color in the Dutch survey. Only $1 \%$ of the subjects did so. Apparently, most subjects were not suspicious and had no preference between the two winning colors. This implies $f_{Y} \sim f_{P}$ and consequently $q=0.5$.

The survey measured the matching probability $r$ for which subjects were indifferent between urn $\mathrm{A}$ and urn $\mathrm{K}$ with $r^{*} 100$ balls of their winning color. Subjects made a series of choices between the two urns to determine $r$, where urn A remained the same while the proportion of winning balls in urn $\mathrm{K}$ changed depending on previous choices.

At the end of the surveys, one randomly selected choice was played for real. A ball was drawn from the urn that the subject preferred in that choice. The subject received 15 euro (dollar in the US sample) if the ball was of his winning color and nothing otherwise.

Figure 1 shows the estimated distribution of $\sigma$ in the two datasets using a kernel density estimate. In the Dutch (US) dataset, the median value of $\sigma$ was equal to 0.05 (0.02), which corresponds with a matching probability of $40.6 \%(47.0 \%)$. Both distributions are centered slightly to the right of zero and concentrated in the ambiguity averse domain. Still, 22.6\% (35.9\%) of subjects were ambiguity seeking. The box at the far left of the distribution shows that $6.2 \%(4.5 \%)$ of the subjects gave matching probabilities close to 1 , which corresponds with a value of $\sigma$ less than $-0.8(-0.6)$. Similarly, the box on the far right indicates that $9.6 \%$ $(3.6 \%)$ gave matching probabilities close to zero, which corresponds with a $\sigma$ value greater than 0.8 .

Table 1 explores whether ambiguity attitudes were correlated with demographic variables. The first and the third column show the correlations between $\sigma$ and the demographic variables, the second and the fourth column the correlations between
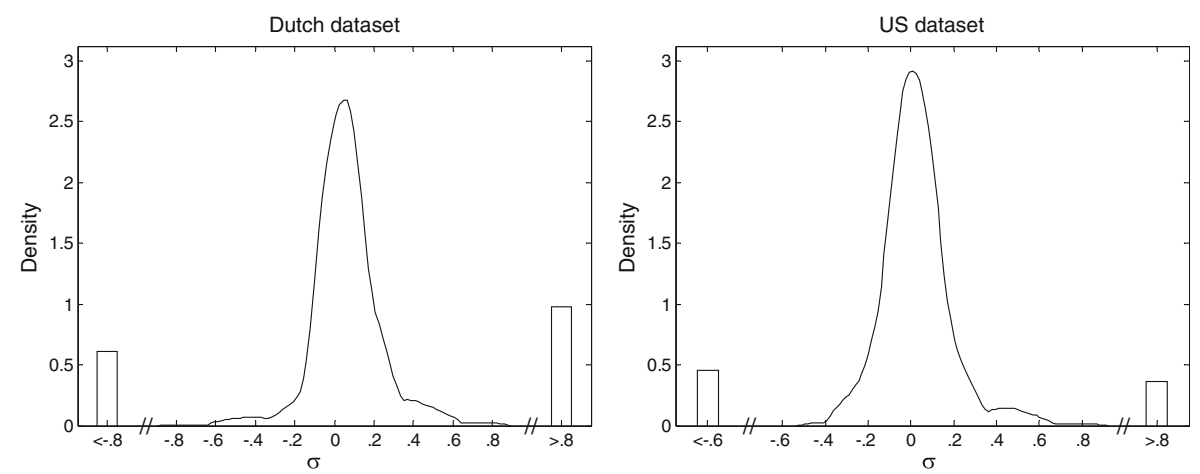

Fig. 1 Kernel density estimates of respondents' $\boldsymbol{\sigma}$ values. The Epanechnikov function was used, with a kernel width of 0.07 . The boxes at the upper and lower end indicate the proportion of subjects with $\sigma$ values greater than 0.8 and less than $-0.8 /-0.6$ 
Table 1 Correlations between demographic variables and ambiguity aversion $(\sigma)$ and deviation from ambiguity neutrality $(|\sigma|)$

\begin{tabular}{lllll}
\hline & Dutch dataset & \multicolumn{3}{l}{ US dataset } \\
& $\sigma$ & $|\sigma|$ & $\sigma$ & $|\sigma|$ \\
\hline Gender (female $=1)$ & 0.012 & -0.002 & $-0.054^{* * *}$ & 0.011 \\
Age & $-0.070^{* * *}$ & $0.142^{* * *}$ & $-0.032^{*}$ & 0.001 \\
High income & -0.003 & $-0.047^{* *}$ & 0.020 & $-0.060^{* * *}$ \\
High education & -0.005 & $-0.106^{* * *}$ & $0.031^{*}$ & $-0.064^{* * *}$ \\
$\mathrm{~N}$ & 1821 & 1821 & 3217 & 3217 \\
\hline
\end{tabular}

*significant at $10 \%$ level, $* * 5 \%, * * * 1 \%$

$|\sigma|$ and the demographic variables. ${ }^{8}$ We also analyzed the correlations with $|\sigma|$ because this indicates the deviations from ambiguity neutrality. Such deviations imply violations of either probabilistic sophistication or dynamic consistency, two conditions that are generally considered normative. Ambiguity neutrality is therefore often perceived as the rational benchmark in choice under uncertainty (e.g., Wakker 2010, p. 326).

In the Dutch sample, the only variable that was correlated with $\sigma$ was age, with older respondents being less ambiguity averse. The second column shows that age was positively correlated with $|\sigma|$, which suggests that older respondents had more extreme ambiguity attitudes. Income and education were negatively correlated with the deviation from ambiguity neutrality, which is consistent with the finding that people with higher cognitive abilities deviate less from models of rational choice (Frederick 2005; Dohmen et al. 2010).

In the US sample, women were less ambiguity averse than men. Older and less educated people were marginally less ambiguity averse. Although age and $|\sigma|$ were uncorrelated, in contrast with what we found in the Dutch dataset, the correlation coefficients for income and education were remarkably similar to their Dutch counterparts. All correlations were negative, indicating that those with higher income and education were closer to ambiguity neutrality.

\section{Concluding remarks}

Hansen and Sargent's (2001) multiplier preferences are a popular model in macroeconomics and finance. In its original form, multiplier preferences only capture ambiguity aversion, which make them less suitable for applications at the micro level where ambiguity seeking is also commonly observed. This paper extends multiplier preferences to include ambiguity seeking and it gives a preference foundation for these extended multiplier preferences. We also show how extended multiplier preferences

\footnotetext{
${ }^{8}$ The coefficients shown are Pearson correlation coefficients. Spearman rank-correlations show qualitatively similar results.
} 
can be measured and thereby obtain an axiomatically-founded measure of ambiguity aversion that can easily be applied in empirical studies and that captures the substantial heterogeneity in ambiguity attitudes that typically exists in micro data. As an illustration, we applied our method to two large scale representative surveys, one from the Netherlands and one from the US. In both samples a substantial fraction of the respondents was ambiguity seeking, which illustrates the desirability of our extension of multiplier preferences. Our data also indicate that better-educated respondents and those with higher incomes were less likely to deviate from the rational benchmark.

Acknowledgements The editor W. Kip Viscusi, an anonymous reviewer, Roy Kouwenberg, Roger Laeven, Tomasz Strzalecki, and Peter P. Wakker gave helpful comments on earlier versions. We thank Christina Stoddard for her careful proofreading. This research was made possible through financial support from the Netherlands Organization for Scientific Research (NWO).

\section{Appendix}

Lemma A1: Preferences $\succcurlyeq$ are extended multiplier preferences if and only if there exists a $\sigma \in \mathbb{R}$ such that $\succcurlyeq$ can be represented by SOEU with $q \in \Delta(S)$ and $\varphi=\varphi_{\sigma}$.

Proof:

The equivalence between robust preferences and $\varphi(t)=-e^{-\sigma t}$ has been shown by Strzalecki (2011). It is based on Proposition 1.4.2 of Dupuis and Ellis (1997) stating that for all countably additive probability measures $q \in \Delta(S)$ and for all $\Sigma$-measurable functions $v$ :

$$
\min _{p \in \Delta(S)} \int_{S} v(s) d p(s)+\frac{1}{\lambda} R(p \| q)=\varphi_{\lambda}^{-1}\left(\int_{S} \varphi_{\lambda}(v(s)) d q(s)\right)
$$

For $\sigma<0$, we apply this formula to $v=-u \circ f$ and $\lambda=-\sigma$ and we obtain:

$$
\begin{aligned}
\max _{p \in \Delta(S)} \int_{S} u(f(s)) d p(s)+\frac{1}{\sigma} R(p \| q) & =-\left[\min _{p \in \Delta(S)} \int_{S} v(s) d p(s)+\frac{1}{\lambda} R(p \| q)\right] \\
& =-\varphi_{\lambda}^{-1}\left(\int_{S} \varphi_{\lambda}(v(s)) d q(s)\right) \\
& =\varphi_{\sigma}^{-1}\left(\int_{S} \varphi_{\sigma}(u(f(s))) d q(s)\right) .
\end{aligned}
$$

The last equality follows from $\varphi_{\sigma}^{-1}(t)=-\frac{\ln (t)}{\sigma}=\frac{\ln (t)}{\lambda}=-\varphi_{\lambda}^{-1}(-t)$ and $\varphi_{\lambda}(v(s))$ $=-e^{-\lambda v(s)}=-e^{-\sigma u(f(s)))}=-\varphi_{\sigma}(u(f(s)))$.

Hence, both robust and opportunity seeking preferences are equivalent to SOEU with an exponential $\varphi$ function.

\section{Proof of Theorem 1:}

(2) $\Rightarrow(1)$. Because (2) is a normalized niveloid that represents $\succcurlyeq$ and $u$ is nonconstant and affine, Lemma 28 in Maccheroni et al. (2006) implies that $\succcurlyeq$ is a continuous, nondegenerate weak order that satisfies weak certainty independence and monotonicity. Because q is countably additive, $\succcurlyeq$ satisfies uniform continuity by Theorem 5.4 in Krantz et al. (1971). 
Finally, by Proposition 1.4.2 in Dupuis and Ellis (1997), (2) is equivalent to a second order expected utility representation. Consequently, the sure thing principle must hold.

We show that $(1) \Rightarrow(2)$ by closely following Strzalecki's proof without imposing uncertainty aversion. First we introduce some new notation. Let $B_{0}(\Sigma)$ denote the set of all real-valued $\Sigma$-measurable simple functions ${ }^{9}$ and let $B_{0}(\Sigma, \mathrm{K})$ denote the set of functions in $B_{0}(\Sigma)$ that take values in a convex set $K \subseteq \mathbb{R}$. Let $\Phi_{3}$ denote the set of finite partitions of $S$ that contain at least three essential events. For all $G \in \Phi_{3}$, let $\mathcal{A}(G)$ be the algebra generated by $G$ and let $\mathcal{F}_{G}$ denote the set of acts in $\mathcal{F}$ that are measurable with respect to $\mathcal{A}(G)$.

By Lemmas 25 and 28 of Maccheroni et al. (2006), there exist a real-valued nonconstant affine function $u$ on $\Delta(Z)$ and a normalized real-valued functional $I: B_{0}(\Sigma, \mathcal{U}) \rightarrow \mathbb{R}$ where $\mathcal{U}$ is the range of $u(\Delta(Z))$ and such that for all acts $f, g \in \mathcal{F}, f \geqslant g$ iff $I(u \circ f) \geq I(u \circ g)$ and $I(\alpha \psi+(1-\alpha) k)=I(\alpha \psi)+(1-\alpha) k$ for all $\psi \in B_{0}(\Sigma, \mathcal{U}), k \in \mathcal{U}$ and $\alpha \in(0,1)$.

Theorem 1 in Grant et al. (2009) ensures that for finite $S \succcurlyeq$ can be represented by $f$ $\longmapsto \sum_{s \in S} v_{S}(u(f(s)))$ with $u$ nonconstant and affine and with range $\mathcal{U}$ and $v_{s}$ continuous, nondecreasing, and with at least three $v_{s}$ nonconstant. Weak certainty independence then ensures that indifference curves in the utility space are parallel and have common supporting hyperplanes at the set of constant vectors in $\mathcal{U}^{S}$. By the proof of Theorem 3 in Grant et al. (2009) it follows that for all $G \in \Phi_{3}$ the restriction of $\succcurlyeq$ to $\mathcal{F}_{G}$ can be represented by $f \longmapsto \sum_{s \in S} p_{G}(s) \varphi_{G}\left(u_{G}\left(f_{s}\right)\right)$ with $u_{G}$ nonconstant and affine, $\varphi_{G}$ continuous and strictly increasing, and measure $p_{G}: \mathcal{A}(\mathrm{G}) \rightarrow[0,1]$ such that at least three events in $\mathrm{G}$ are nonzero. In applying Theorem 3 of Grant et al. (2009), we replace uncertainty aversion and their Axiom A.7 by weak certainty independence. Uncertainty aversion is used in the application of Theorem 3 in Debreu and Koopmans (1982) to derive differentiability of the functions $v_{s}$. However, as noted by Grant et al. (2009) and Maccheroni et al. (2006 ,p.1475, 1491), weak certainty independence implies Lipschitz continuity and hence absolute continuity of the $v_{s}$ functions so that they can be represented as integrals of their (almost everywhere) derivatives. Because we no longer impose uncertainty aversion, $\varphi$ need not be concave as in Theorem 3 of Grant et al. (2009). By Theorem 4 in Strzalecki (2011), $\succcurlyeq$ can be represented by second order expected utility $f \mapsto \int_{S} \varphi\left(u\left(f_{s}\right)\right) d q(s)$ with $q \in \Delta(Z)$ and $\varphi$ continuous and strictly increasing. Strzalecki (2011) also imposed uncertainty aversion but he only requires this to derive concavity of $\varphi$, which we do not impose. We only require $\varphi$ to be nonconstant, which follows from monotonicity. $q$ is countably additive by uniform continuity (Villegas 1964, Theorem 1). Moreover, if $(u, \varphi, q)$ and $\left(u^{\prime}, \varphi^{\prime}, q^{\prime}\right)$ both represent $\succcurlyeq$ then there exist $\alpha, A>0, \beta, B \in \mathbb{R}$ such that $q^{\prime}=q, u^{\prime}=\alpha u+\beta, \varphi^{\prime}(\alpha r+\beta)=A \varphi(r)+B$ for all $r$ in $\mathcal{U}$.

$I$ represents $\succcurlyeq$ and is translation invariant, i.e. for all $f, g \in \mathcal{F}$ and $k$ such that $f(s)$ $+k, g(s)+k \in \mathcal{U}$ for all $s \in S, I(u \circ f) \geq I(u \circ g)$ iff $I(u \circ f+k)=I(u \circ f)+k \geq I(u \circ g)+$ $k=I(u \circ g+k)$. It then follows that for all acts $f, g \in \mathcal{F}$ and $k$ such that $f(s)+k, g(s)$ $+k \in \mathcal{U}$ for all $s \in S, \int_{S} \varphi(u(f(s))) d q(s) \geq \int_{S} \varphi(u(g(s))) d q(s)$ iff $\int_{S} \varphi(u(f(s)+k) d q$ $(s) \geq \int_{S} \varphi(u(g(s)+k) d q(s)$.

Hence, $(u, \varphi, q)$ and $\left(u, \varphi_{k}, q\right)$ defined by $\varphi_{k}(l)=\varphi(l+k), \forall l, l+k \in \mathcal{U}$ are both SOEU representations of $\succcurlyeq$. Consequently, $\varphi(l+k)=A(k) \varphi(l)+B(k)$. Because $\varphi$ is nonconstant, if $\mathcal{U}$ is unbounded, it follows from Corollary 1 in Aczél (1966,

\footnotetext{
${ }^{9}$ A function is simple if it takes no more than countably many distinct values.
} 
Section 3.1.3) that $\varphi$ equals $\varphi_{\sigma}$. If $\mathcal{U}$ is bounded then because $\varphi$ is nonconstant Theorem 4 in Aczél (2005) implies $\varphi=\varphi_{\sigma}$ on the interior of $\mathcal{U}$. Because $\varphi$ is continuous, the extension to all of $\mathcal{U}$ follows.

By Proposition 1.4.2 in Dupuis and Ellis (2011) and Lemma A1, we then obtain the extended multiplier representation.

\section{Proof of Observation 1:}

The proof of Theorem 1 already showed that the probability measure $q$ is unique and that the utility function $u$ is unique up to positive affine transformations. We also know that for $A>0$ and $B \in \mathbb{R}, \varphi^{\prime}=A \varphi+B$. Because $e^{-\sigma^{\prime} u^{\prime}}=e^{-\sigma^{\prime}(\alpha u+\beta)}=e^{-\sigma^{\prime} \beta} e^{-\alpha \sigma^{\prime} u}$, it follows from the uniqueness properties of $\varphi$ that $\sigma^{\prime}=\frac{1}{\alpha} \sigma$.

\section{Proof of Theorem 2:}

Ambiguity aversion states that preferences are convex. Hence it is equivalent to a concave representation. Since $u$ is linear with respect to mixture of lotteries, ambiguity aversion is equivalent to SOEU with $\varphi$ concave, which means $\sigma \geq 0$. The opposite reasoning applies to ambiguity seeking.

\section{Proof of Theorem 3:}

(2) $\Rightarrow$ (1) is trivial. Assume (1). It implies $u_{1} \approx u_{2}$ (Ghirardato and Marinacci 2002, Proposition 11). We scale utility such that $u_{1}=u_{2}$. Recode lotteries into expected utilities. Using the second-order expected utility formulation of extended multiplier preferences and the results of Yaari (1969), we immediately obtain $q_{1}=q_{2}$ and $\varphi_{2}$ more concave than $\varphi_{1}$, which implies $\sigma_{1} \leq \sigma_{2}$.

Proof that there is a unique solution $\sigma$ for each value of $r$.

$f_{Y} \sim f_{P}$ and $f_{P} \sim 15_{r} 0$ jointly imply $\varphi_{\sigma}(15 r)=1 / 2 \varphi_{\sigma}(15)+1 / 2 \varphi_{\sigma}(0)$, which is equivalent to $15 r=1 / 2(15)+1 / 2(0)$ if $\sigma=0$ and to $\exp (-15 \sigma r)=1 / 2 \exp (-15 \sigma)+1 / 2 \exp (0)$ otherwise. Hence,

$$
\begin{aligned}
& r=1 / 2 \text { if } \sigma=0 \\
& r=-\frac{\ln (\xi \exp (-15 \sigma)+\xi)}{15 \sigma} \text { if } \sigma \neq 0
\end{aligned}
$$

The proof that $r$ is continuous and decreasing as a function of $\sigma$ is elementary. By the intermediate value theorem, there is a unique solution $\sigma$ for each $r \in(0,1)$.

Open Access This article is distributed under the terms of the Creative Commons Attribution 4.0 International License (http://creativecommons.org/licenses/by/4.0/), which permits unrestricted use, distribution, and reproduction in any medium, provided you give appropriate credit to the original author(s) and the source, provide a link to the Creative Commons license, and indicate if changes were made.

\section{References}

Aczél, J. (1966). Lectures on functional equations and their applications. New York: Academic Press.

Aczél, J. (2005). Utility of extension of functional equations-When possible. Journal of Mathematical Psychology, 49(6), 445-449.

Cerreia-Vioglio, S., Maccheroni, F., Marinacci, M., \& Montrucchio, L. (2012). Probabilistic sophistication, second order stochastic dominance and uncertainty aversion. Journal of Mathematical Economics, 48, 271-283. 
Debreu, G., \& Koopmans, T. C. (1982). Additively decomposed quasiconvex functions. Mathematical Programming, 24(1), 1-38.

Dillenberger, D., \& Segal, U. (2015). Recursive ambiguity and Machina's examples. International Economic Review, 56(1), 55-61.

Dimmock, S. G., Kouwenberg, R., Mitchell, O. S., \& Peijnenburg, K. (2015). Estimating ambiguity preferences and perceptions in multiple prior models: Evidence from the field. Journal of Risk and Uncertainty, 51(3), 219-244.

Dimmock, S. G., Kouwenberg, R., Mitchell, O. S., \& Peijnenburg, K. (2016a). Ambiguity aversion and household portfolio choice puzzles: Empirical evidence. Journal of Financial Economics, 119(3), 559-577.

Dimmock, S. G., Kouwenberg, R., \& Wakker, P. P. (2016b). Ambiguity attitudes in a large representative sample. Management Science, 62(5), 1363-1380.

Dohmen, T., Falk, A., Huffman, D., \& Sunde, U. (2010). Are risk aversion and impatience related to cognitive ability? American Economic Review, 100(3), 1238-1260.

Dupuis, P., \& Ellis, R. S. (1997). A weak convergence approach to the theory of large deviations. New York: Wiley.

Ellsberg, D. (1961). Risk, ambiguity and the Savage axioms. Quarterly Journal of Economics, 75(4), 643-669.

Frederick, S. (2005). Cognitive reflection and decision making. Journal of Economic Perspectives, 19(4), $25-42$.

Ghirardato, P., \& Marinacci, M. (2002). Ambiguity made precise: A comparative foundation. Journal of Economic Theory, 102(2), 251-289.

Gilboa, I., \& Marinacci, M. (2013). Ambiguity and the Bayesian paradigm. In D. Acemoglu, M. Arellano, \& E. Dekel (Eds.), Advances in economics and econometrics, tenth world congress, volume I, economic theory. New York: Cambrige University Press.

Grant, S., \& Polak, B. (2013). Mean-dispersion preferences and constant absolute uncertainty aversion. Journal of Economic Theory, 148(4), 1361-1398.

Grant, S., Polak, B., \& Strzalecki, T. (2009). Second-order expected utility. SSRN: http://dx.doi.org/10.2139 /ssrn.2328936.

Hansen, L. P., \& Sargent, T. J. (2001). Robust control and model uncertainty. American Economic Review, 91(2), 60-66.

Klibanoff, P., Marinacci, M., \& Mukerji, S. (2005). A smooth model of decision making under ambiguity. Econometrica, 73(6), 1849-1892.

Krantz, D. H., Luce, R. D., Suppes, P., \& Tversky, A. (1971). Foundations of measurement: Vol. 1: Additive and polynomial representations. New York: Academic Press.

Maccheroni, F., Marinacci, M., \& Rustichini, A. (2006). Ambiguity aversion, robustness, and the variational representation of preferences. Econometrica, 74(6), 1447-1498.

Machina, M. J. (2009). Risk, ambiguity, and the rank-dependence axioms. American Economic Review, 99(1), 385-392.

Machina, M. J. (2014). Ambiguity aversion with three or more outcomes. American Economic Review, 104(12), 3814-3840.

Machina, M. J., \& Siniscalchi, M. (2014). Ambiguity and ambiguity aversion. In Handbook of the economics of risk and uncertainty (Vol. 1, 729-807). Amsterdam: Elsevier. http://dx.doi.org/10.1016/B978-0-44453685-3.00013-1

Nau, R. F. (2006). Uncertainty aversion with second-order utilities and probabilities. Management Science, 52(1), 136-145.

Neilson, W. S. (2010). A simplified axiomatic approach to ambiguity aversion. Journal of Risk and Uncertainty, 41(2), 113-124.

Pratt, J. W. (1964). Risk aversion in the small and in the large. Econometrica, 32(1-2), 122-136.

Schmeidler, D. (1989). Subjective probability and expected utility without additivity. Econometrica, 57(3), 571-587.

Segal, U. (1987). The Ellsberg paradox and risk aversion: An anticipated utility approach. International Economic Review, 28, 175-202.

Strzalecki, T. (2011). Axiomatic foundations of multiplier preferences. Econometrica, 79(1), 47-73.

Trautmann, S. T., \& van de Kuilen, G. (2015). Ambiguity attitudes. In G. Keren \& G. Wu (Eds.), The WileyBlackwell handbook of judgment and decision making (pp. 89-116). West Sussex: Wiley.

Villegas, C. (1964). On qualitative probability $\sigma$-algebras. Annals of Mathematical Statistics, 35(4), 1787-1796.

Viscusi, W. K., \& Magat, W. A. (1992). Bayesian decisions with ambiguous belief aversion. Journal of Risk and Uncertainty, 5(4), 371-387.

Wakker, P. P. (1989). Additive representations of preferences: A new Foundation of Decision Analysis. Dordrecht: Kluwer Academic Publishers.

Wakker, P. P. (2010). Prospect theory for risk and ambiguity. Cambridge, UK: Cambridge University Press.

Yaari, M. E. (1969). Some remarks on measures of risk aversion and on their uses. Journal of Economic Theory, 1(3), 315-329. 